УДК 681.513 .5

\title{
THE PROBLEM OF THE OPTIMAL MINIMAX LIMIT CONTROL OF OBJECTS WITH DISTRIBUTED PARAMETERS
}

\author{
O. Lobok, N. Savizkaia, B. Goncharenko \\ National University of Food Technologies \\ M. Sych \\ National University of Bioresources and Natural Resources of Ukraine
}

$\quad$ Key words:
Minimax control
Regulators
Distributed parameter
systems
Optimization
Gradient projection
method
Point and mobile limit
control

Article history:

Received 13.07.2018

Received in revised form

31.07.2018

Accepted 20.08.2018

Corresponding author:

B. Goncharenko

E-mail:

GoncharenkoBN@i.ua

\section{ABSTRACT}

The problem of minimax control synthesis for objects that are described by a two-dimensional heat conduction equation of parabolic type is solved in the paper. It is assumed that the control object functions under uncertainty conditions, and the perturbations acting on the object belong to some given hyperelipsoid. The problem of constructing a regulator in the state of an object for cases of point and mobile limit control is considered in accordance with the integral-quadratic quality criterion. With the help of numerical optimization methods, the problem of determining the optimal location of concentrated regulators at the boundary of a rectangular region and the problem of finding the optimal law of motion of a mobile limit regulator is solved. The problem is posed and solved in the minimax formulation when there is an optimal control on the state of the object functioning under uncertainty conditions so that the regulator minimizes the maximum control error from a set of possible values, taking into account the most unfavorable perturbations that can act on the object or system. In this case, the perturbations of the object belong to a given limited region. The results of computational experiments illustrating the effectiveness of the constructed limiting concentrated and moving regulators are presented. The obtained results indicate that the controls found in the work are indeed optimal and ensure minimum errors (deviations from the given state) of the functioning of the system and energy costs for the implementation of control under given conditions and in the absence of any information on external action other than the region of permissible perturbations.

In the work, for the first time, a minimax approach was used to control the objects described by the two-dimensional parabolic type thermal conductivity equation; the theoretical positions of synthesis of minimax regulators for cases of lumped boundary (point) and moving regulators are considered; algorithmic software is developed that allows to simulate the dynamics of the constructed minimax-regulators and to investigate the corresponding transients.

DOI: $10.24263 / 2225-2924-2018-24-4-4$ 


\title{
ЗАДАЧА ОПТИМАЛЬНОГО МІНІМАКСНОГО ГРАНИЧНОГО КЕРУВАННЯ ОБ'ЄКТАМИ 3 РОЗПОДІЛЕНИМИ ПАРАМЕТРАМИ
}

\author{
О.П. Лобок, Н.М. Савицька, Б.М. Гончаренко \\ Національний університет харчових технологій
} М.А. Сич

Національний університет біоресурсів і природокористування Украйни

У статті розв'язується задача синтезу мінімаксного керування для об'єктів, які описуються двовимірним рівнянням теплопровідності параболічного типу. Передбачається, що об'єкт керування функиіонує в умовах невизначеності, причому збурення, що діють на об'єкт, належсать до заданого гіпереліпсоїду. Розглядається задача побудови регулятора за станом об'єкта для випадків точкового та рухомого граничного керування згідно з інтегрально-квадратичним критерієм якості. За допомогою числових оптимізаційних методів розв'язана задача визначення оптимального розтамування зосереджених регуляторів на границі прямокутної області та задача пошуку оптимального закону переміщення рухомого граничного регулятора. Задача ставиться та розв'язується в мінімаксній постановиі, тобто знаходиться оптимальний регулятор за станом об'єкта, який функиіонує в умовах невизначеності так, щчо він передбачає мінімізацію максимальної похибки (відхилення поточного стану системи від заданого або бажаного) з множини можливих значень з урахуванням найбільш несприятливих збурень, щчо можуть діяти на об'єкт або систему. Причому збурення об 'єкта належать до заданої обмеженої області. Наводяться результати обчислювальних експериментів, які ілюструють ефективність побудованих зосереджених граничних (точкових) $i$ рухомих регуляторів. Отримані результати свідчать про те, щзо знайдені керування дійсно є оптимальними $і$ забезпечують мінімум похибки (відхилення від заданого стану) функиіонування системи та енергетичних затрат на здійснення керування при заданих умовах і відсутності будь-якої інформачії про зовнішній вплив, крім області допустимих збурень.

Уперше застосовано мінімаксний підхід для керування об'єктами, які описуються двовимірним рівнянням теплопровідності параболічного типу; розглянуто теоретичні положення синтезу мінімаксних регуляторів для випадків зосереджених граничних (точкових) і рухомих регуляторів; розроблено алгоритмічно-програмне забезпечення, яке дає змогу моделювати динаміку побудованих мінімаксних регуляторів і досліджувати відповідні перехідні процеси.

Ключові слова: мінімаксне керування, регулятори, системи з розподіленими параметрами, оптимізачія, метод проекиї градієнта, точкове та рухоме граничне керування.

Постановка проблеми. У зв'язку з широким розповсюдженням нових прогресивних технологій, пов'язаних з використанням електронних, іонних, 
лазерних та інших випромінювань, останніми роками почалось інтенсивне вивчення можливостей оптимального керування розподіленими системами джерел шляхом зміни розташування точкових джерел випромінювань i законів пересування рухомих.

Постановка задач точкового і рухомого керування, деякі методи їх розв'язання наведені в [1-3]. Однією 3 дуже важливих і складних задач є вибір оптимальної стратегії точкового і рухомого керування для систем, що функціонують в умовах невизначеності. Саме цій проблемі і присвячена ця пропонована стаття, в якій розв'зуються задачі вибору оптимального розташування точкових регуляторів і пошуку оптимального закону руху (переміщення) рухомого джерела на границі прямокутної області для процесу теплоперенесення, перебіг якого відбувається в умовах неповної інформації. Теорія керування рухається в напрямку ускладнення досліджуваних явищ, процесів і зменшення інформації про систему керування, об'єкт, його особливості, властивості, характеристики, умови функціонування, зовнішні впливи. Враховуючи все вищезазначене, обраний напрям досліджень є перспективним і має високий рівень актуальності.

Мета статті: практичне застосування теорії синтезу мінімаксних регуляторів для задач керування вибором оптимального розташування точкових регуляторів і пошуку оптимального закону руху в умовах невизначеності зовнішніх збурень, що діють на них, крім області їхньої допустимості, а також дослідження ефективності запропонованих регуляторів за допомогою обчислювальних експериментів.

Викладення основних результатів дослідження. У багатьох технологічних об'єктах автоматизації наявні дифузійні процеси або теплоперенесення. Так, процес теплоперенесення в однорідній тонкій прямокутній пластині описується функцією $\varphi(x, t)$, яка в області $Q_{T}=\Omega \cdot(0, T)$, де $\Omega=\left\{\left(x_{1}, x_{2}\right): 0<x_{1}<l_{1}, 0<x_{2}<l_{2}\right\}, l_{1}, l_{2}>0, T<\infty$, задовольняє рівняння:

$$
\frac{\partial \varphi(x, t)}{\partial t}=a \Delta_{x} \varphi(x, t)+f_{1}(x, t),(x, t) \in Q_{T}
$$

а на границі $Q_{T}$ — ще й додаткові умови:

$$
\varphi(x, 0)=f_{0}(x), x \in \Omega ; \varphi(x, t)=\sum_{i=1}^{N} \delta\left(x-v_{i}(t)\right) u_{i}(t),(x, t) \in \Gamma \cdot(0, T) .
$$

Тут $\Delta_{x}=\frac{\partial^{2}}{\partial x_{1}^{2}}+\frac{\partial^{2}}{\partial x_{2}^{2}}-$ двовимірний оператор Лапласа; $a>0-$ коефіцієнт температуропровідності; $\Gamma$ - границя прямокутної області $\Omega ; \delta(x-y)-$ дельта-функція Дірака; $t \rightarrow v_{i}(t) \in \Gamma$ - вимірні функції, що задають рух граничних джерел; $u_{i}(t) \in L_{2}(0, T)$ - функції керування; $f_{0}(x) \in L_{2}(\Omega)$, $f_{1}(x, t) \in L_{2}\left(Q_{T}\right)$ - невідомі функції, що належать до області:

$$
S_{t}=\left\{\left(f_{0}, f_{1}\right): G\left(f_{0} ; f_{1}(\tau), 0<\tau<t\right) \leq 1\right\}, t \in(0, T],
$$


де

$$
G\left(f_{0} ; f_{1}(\tau), 0<\tau<t\right)=F_{0} \int_{\Omega} f_{0}^{2}(x) d x+F_{1} \int_{0}^{t} \int_{\Omega} f_{1}^{2}(x, \tau) d x d \tau,
$$

$F_{0}, F_{1}$ - додатні постійні величини, що відображають внесок перешкод; $f_{0}$ i $f_{1}(t)$ в результуюче збурення, що діє на систему (1), (2).

Під розв'язком крайової задачі (1), (2) будемо розуміти таку функцію $\varphi(x, t) \in L_{2}\left(Q_{T}\right)$, яка задовольняє таку інтегральну тотожність:

$$
\begin{gathered}
-\int_{0}^{T} \int_{\Omega} \varphi(x, t)\left(\frac{\partial \eta(x, t)}{\partial t}+a \Delta_{x} \eta(x, t)\right) d x d t=\int_{\Omega} f_{0}(x) \eta(x, 0) d x+\int_{0}^{T} \int_{\Omega} f_{1}(x, t) \eta(x, t) d x d t- \\
-\left.a \sum_{i=1}^{N} \int_{0}^{T} u_{i}(t) \frac{\partial \eta(x, t)}{\partial n}\right|_{x=v_{i}(t)} d t \quad \forall \eta(x, t) \in \Phi
\end{gathered}
$$

де $\partial / \partial n$ - похідна по зовнішній нормалі $\vec{n}$ до границі $\Gamma$ області $\Omega$, $\Phi=\left\{\eta(x, t): \eta(x, t) \in H^{3,1}\left(Q_{T}\right), \eta(x, T)=0, x \in \Omega ; \eta(x, t)=0,(x, t) \in \Gamma \cdot(0, T)\right\}$, $H^{3,1}\left(Q_{T}\right)$ - соболівський простір [4].

Можна показати [5], що розв'язок рівняння (4) при заданих керуваннях $u_{i}(t) \in L_{2}(0, T)$ існує і $є$ єдиним в просторі $L_{2}\left(Q_{T}\right)$.

Задача вибору оптимальної стратегії мінімаксного керування [6;8] буде полягати в тому, щоб знайти вектор-функції $v^{*}(t)=\left[v_{1}^{*}(t), v_{2}^{*}(t), \ldots, v_{N}^{*}(t)\right]^{T}$ i $u^{*}(t)=\left[u_{1}^{*}(t), u_{2}^{*}(t), \ldots, u_{N}^{*}(t)\right]^{T}$ з умови

$$
I\left(u^{*}, v^{*}\right)=\inf _{v} \inf _{u} I(u, v),
$$

де

$$
I(u, v)=\sup _{S_{T}}\left[\int_{\Omega} S(x) \varphi(x, T) d x\right]^{2}+\int_{0}^{T} \sum_{i=1}^{N} d_{i} \sup _{S_{t}} u_{i}^{2}(t) d t,
$$

$S(x) \in L_{2}(\Omega), d_{i}=$ const $>0, i=1,2, \ldots, N$, при заданій структурі керування $u_{i}(t)$ у вигляді лінійного зворотного зв'язку виду:

$$
u_{i}(t)=\int_{\Omega} R_{i}(x, t) \varphi(x, t) d x .
$$

Розв’язування сформульованої задачі будемо здійснювати в два етапи: спочатку розв'яжемо задачу визначення оптимального керування $u^{*}(t)$ з умови

$$
I\left(u^{*}, v\right)=\inf _{u} I(u, v)
$$

при фіксованій вектор-функції $v(t)$, а потім знайдемо $v^{*}(t)$, при якому 


$$
I\left(u^{*}, v^{*}\right)=\inf _{v} I\left(u^{*}, v\right) .
$$

Використовуючи результати, наведені у [7; 8], можна довести справедливість такого твердження: оптимальне керування $u^{*}(t)$ оптимізаційної задачі (1), (2), (6), (7), що задовольняє необхідні умови оптимальності має вигляд:

$$
u_{i}^{*}(t)=\int_{\Omega} R_{i}^{*}(x, t) \varphi(x, t) d x, R_{i}^{*}(x, t)=a d_{i}^{-1} \alpha^{-1}(t) g(x, t) h\left(v_{i}(t), t\right),
$$

де

$$
\begin{gathered}
\alpha(t)=1+a^{2} \sum_{k=1}^{N} d_{k}^{-1} \int_{t}^{T} h^{2}\left(v_{k}(\tau), \tau\right) d \tau, \\
{\left[\begin{array}{l}
g(x, t) \\
h(x, t)
\end{array}\right]=\sum_{i=1}^{\infty} s_{i} e^{\lambda_{i}(t-T)}\left[\begin{array}{c}
\omega_{i}(x) \\
r_{i}(x)
\end{array}\right], s_{i}=\int_{\Omega} S(x) \omega_{i}(x) d x .}
\end{gathered}
$$

У співвідношенні (11) $i=\left(i_{1}, i_{2}\right)-$ мультиіндекс,

$$
r_{i}(x)=r_{i_{1}, i_{2}}\left(x_{1}, x_{2}\right)=\frac{2 \pi}{\sqrt{l_{1} l_{2}}} \begin{cases}(-1)^{i_{1}} \frac{i_{1}}{l_{1}} \sin \frac{\pi i_{2} x_{2}}{l_{2}}, & x_{1}=l_{1}, \\ -\frac{i_{1}}{l_{1}} \sin \frac{\pi i_{2} x_{2}}{l_{2}}, & x_{1}=0, \\ (-1)^{i_{2}} \frac{i_{2}}{l_{2}} \sin \frac{\pi i_{1} x_{1}}{l_{1}}, & x_{2}=l_{2}, \\ -\frac{i_{2}}{l_{2}} \sin \frac{\pi i_{1} x_{1}}{l_{1}}, & x_{2}=0, \\ x=\left(x_{1}, x_{2}\right) \in \Gamma, & \end{cases}
$$

де $\lambda_{i}, \omega_{i}(x)$ - власні значення і відповідні ортонормовані в просторі $L_{2}(\Omega)$ власні функції крайової задачі (1), (2), що мають вигляд:

$$
\begin{gathered}
\lambda_{i}=\lambda_{i_{1}, i_{2}}=a \pi^{2}\left[\left(i_{1} / l_{1}\right)^{2}+\left(i_{2} / l_{2}\right)^{2}\right], \\
\omega_{i}(x)=\omega_{i_{1}, i_{2}}\left(x_{1}, x_{2}\right)=\frac{2}{\sqrt{l_{1} l_{2}}} \sin \frac{\pi i_{1} x_{1}}{l_{1}} \sin \frac{\pi i_{2} x_{2}}{l_{2}} .
\end{gathered}
$$

Значення функціоналу (6) на оптимальному керуванні (10) при цьому визначається за формулою:

$$
I\left(u^{*}, v\right)=\frac{W(0)}{F_{0} \alpha(0)}+\frac{1}{F_{1}} \int_{0}^{T} \frac{W(t)}{\alpha(t)} d t,
$$


де

$$
W(t)=\sum_{i=1}^{\infty} s_{i} e^{2 \lambda_{i}(t-T)} .
$$

Перейдемо тепер до розв’язування оптимізаційної задачі (9), (12).

Розглянемо спочатку більш простий випадок, коли $v_{i}(t) \equiv z_{i} \in \Gamma, i=$ $=1,2, \ldots, N$, тобто розв'яжемо задачу оптимального розташування точкових граничних керувань (10). Введемо позначення $z=\left[z_{1}, z_{2}, \ldots, z_{N}\right]^{T}, J(z)=I\left(u^{*}, z\right) \equiv$ $\equiv I\left(u^{*}, v\right)$. Тоді розглядувана задача буде полягати в тому, щоб знайти вектор $z=\left[z_{1}^{*}, z_{2}^{*}, \ldots, z_{N}^{*}\right]^{T}$, при якому

$$
J\left(z^{*}\right)=\inf _{z \in \Omega_{z}} J(z)
$$

де $\Omega_{z}=\left\{z: z=\left[z_{1}, z_{2}, \ldots, z_{N}\right]^{T}, z_{i}=\left(z_{1 i}, z_{2 i}\right) \in \Gamma, i=1,2, \ldots, N ; z_{i} \neq z_{j}, i \neq j\right\}$.

Враховуючи, що функція $J(z) \in$ неперервно диференційованою функцією своїх аргументів, для розв'язання оптимізаційної задачі (14) скористаємось методом проекції градієнта [9]:

$$
z^{k+1}=\operatorname{Pr}_{\Omega_{z}}\left[z^{k}-\rho_{k} \nabla_{z} J\left(z^{k}\right)\right], k=0,1,2, \ldots
$$

де $\operatorname{Pr}_{\Omega_{z}}[z]=\left[y_{1}, y_{2}, \ldots, y_{N}\right]^{T}, y_{i}=\operatorname{Pr}_{\Gamma}\left[z_{i}\right]$ - проекція точки $z_{i}$ на границю $Г$ прямокутної області $\Omega ; z^{k}=\left[z_{1}^{k}, z_{2}^{k}, \ldots, z_{N}^{k}\right]^{T}-$ наближений розв'язок, отриманий на $k$-й ітерації; $z^{0}-$ початкове наближення; $\rho_{k}-$ крок спуску, який обирається з умови монотонного спадання функції мети $J(z)$ [9]; градієнт $\nabla_{z} J(z)$ визначається за формулою:

$$
\begin{gathered}
\nabla_{z} J(z)=\left[\nabla_{z_{1}} J(z), \nabla_{z_{2}} J(z), \ldots, \nabla_{z_{N}} J(z)\right]^{T}, \\
z=\left[z_{1}, z_{2}, \ldots, z_{N}\right]^{T}, z_{i}=\left(z_{1 i}, z_{2 i}\right) \in \Gamma, i=1,2, \ldots, N, \\
\nabla_{z_{n}} J(z)=-2 a^{2}\left[\frac{W(0) \theta_{n}(0)}{F_{0} \alpha^{2}(0)}+\frac{1}{F_{1}} \int_{0}^{T} \frac{W(t) \theta_{n}(t)}{\alpha^{2}(t)} d t\right], \\
\theta_{n}(t)=d_{n}^{-1} \sum_{i=1}^{\infty} \sum_{j=1}^{\infty} \frac{1-e^{\left(\lambda_{i}+\lambda_{j}\right)(t-T)}}{\lambda_{i}+\lambda_{j}} s_{i} s_{j} r_{i}\left(z_{n}\right) P_{j}\left(z_{n}\right),
\end{gathered}
$$

де $P_{j}\left(z_{n}\right)=\left[P_{j}^{1}\left(z_{n}\right), P_{j}^{2}\left(z_{n}\right)\right]$,

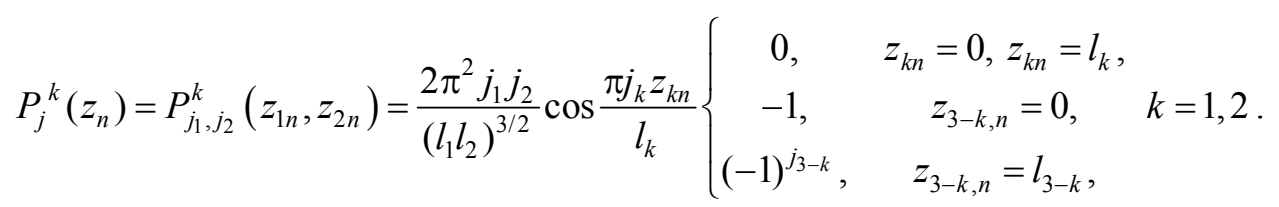


Умова зупинки приймалася у вигляді $\left|J\left(z^{k+1}\right)-J\left(z^{k}\right)\right|<\varepsilon$, де $\varepsilon>0-$ задана точність розв'язку.

Програма може бути реалізована на сучасній алгоритмічній мові при таких початкових даних: $l_{1}=2,0, l_{2}=1,0, T=2,0, F_{0}=0,25, F_{1}=2,0, d_{i}=1,0$, $i=1,2, \ldots, N, S(x)=1,0, \varepsilon=0,001, \rho_{0}=0,8$, число регуляторів $N=5$, за значення коефіцієнта теплопровідності $а$ було взято 0,4 , що відповідає коефіцієнту теплопровідності мідної пластини. Розмірність всіх величин задана в системі [метр, час, град. ${ }^{\circ} \mathrm{C}$, ккал.]. Нескінченні ряди (11), (13) обривались кінцевими сумами, що складались із трьох перших членів. При числовому моделюванні оптимальних керувань $u_{i}^{*}(t)$ припускалось, що збурення $f_{0}(x)$ і $f_{1}(x, t)$ дорівнюють:

$$
f_{0}\left(x_{1}, x_{2}\right)=2 \sin \frac{\pi x_{1}}{l_{1}} \sin \frac{\pi x_{2}}{l_{2}}, f_{1}\left(x_{1}, x_{2}, t\right)=t \sin \frac{\pi x_{1}}{l_{1}} \cos \frac{\pi x_{2}}{l_{2}} .
$$

Відзначимо, що ці збурення є допустимими, оскільки

$$
G\left(f_{0}, f_{1}(\tau), 0<\tau<t\right)=F_{0} l_{1} l_{2}+\frac{1}{12} F_{1} l_{1} l_{2} t^{3}=0,5+\frac{1}{3} t^{3}<1, \forall t \in(0,0,2)
$$

$\mathrm{i}$, як наслідок, $f_{0}(x)$ та $f_{1}(x, t)$ належать до області (3).

$\mathrm{y}$ табл. 1. дано початкове розташування $z^{0}=\left[z_{1}^{0}, z_{2}^{0}, \ldots, z_{N}^{0}\right]^{T}$ точкових граничних регуляторів. Значення функції $J(z)$ при такому розташуванні керувань дорівнює $J\left(z^{0}\right)=0,975632$. Оптимальне розташування регуляторів $z^{*}=\left[z_{1}^{*}, z_{2}^{*}, \ldots, z_{N}^{*}\right]^{T}$, отримане за алгоритмом (15), подано в табл. 2, причому $J\left(z^{*}\right)=0,571874$.

\section{Таблиия 1}

\begin{tabular}{|c|c|c|}
\hline$k$ & $z_{1 k}^{0}$ & $z_{2 k}^{0}$ \\
\hline 1 & 2,0 & 0,0 \\
\hline 2 & 1,0 & 0,0 \\
\hline 3 & 0,0 & 0,0 \\
\hline 4 & 0,0 & 0,5 \\
\hline 5 & 0,0 & 1,0 \\
\hline
\end{tabular}

\section{Таблиия 2}

\begin{tabular}{|c|c|c|}
\hline$k$ & $z_{1 k}^{*}$ & $z_{2 k}^{*}$ \\
\hline 1 & 1,349 & 0,0 \\
\hline 2 & 1,0 & 0,0 \\
\hline 3 & 0,651 & 0,0 \\
\hline 4 & 0,0 & 0,5 \\
\hline 5 & 0,651 & 1,0 \\
\hline
\end{tabular}

На рис. 1 зображені графіки оптимальних точкових керувань (10), що оптимально розташовані на границі $\Gamma$ області $\Omega$ в точках $z_{i}^{*} \in \Gamma, i=1,2, \ldots, N$.

Повернемось тепер до оптимізаційної задачі (9), (12). Розлянемо для простоти одне $(N=1)$ рухоме джерело і нехай збурення $f_{1}(x, t)$ в правій частині рівняння (1) будуть відсутні. Позначимо $u(t)=u_{1}(t), v(t)=v_{1}(t)$, $d=d_{1}, J(v)=I\left(u^{*}, v\right)$. Тоді задача мінімізації функціонала 


$$
J(v)=W(0)\left(F_{0} \alpha(0)\right)^{-1}
$$

еквівалентна наступній оптимізаційній задачі:

$$
L(v)=\int_{0}^{T} h(v(\tau), \tau) d \tau \rightarrow \sup _{\substack{t \rightarrow v(t) \in \Gamma \\ t \in(0, T)}}
$$

де $\alpha(t), h(x, t), W(t)$ - функції, що визначаються за формулами (11), (13).

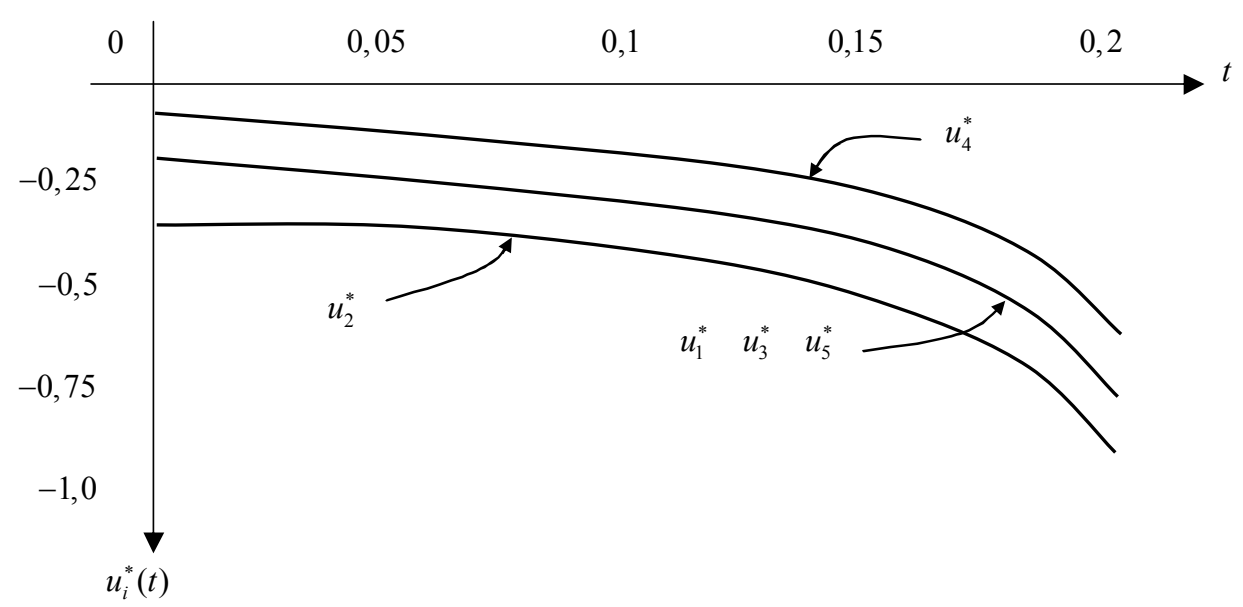

Рис. 1. Графіки оптимальних граничних точкових керувань

Для розв'язання останньої задачі також використовувався метод проекції градієнта виду:

$$
v^{k+1}(t)=\operatorname{Pr}_{\Gamma}\left[v^{k}(t)+\rho_{k} \delta\left[L\left(v^{k}\right) ; t\right]\right], t \in(0, T), k=0,1,2, \ldots,
$$

де $v^{0}(t)$ - початкове наближення; $v^{k}(t)$ - наближений розв'язок, отриманий на $k$-му кроці; $\rho_{k}$ - крок спуску до точки мінімуму; $\delta[L(v) ; t]-$ градієнт Фреше функціонала $L(v)$, який обчислюється за формулою:

$$
\delta[L(v) ; t]=2 h(v(t), t) \rho(v(t), t), \rho(x, t)=\sum_{i=1}^{\infty} s_{i} e^{\lambda_{i}(t-T)} P_{i}(x) .
$$

Алгоритм зупиняється, коли виконується умова $\left|L\left(v^{k+1}\right)-L\left(v^{k}\right)\right|<\varepsilon$, де $\varepsilon>0-$ задана точність розв'язку.

Числова реалізація алгоритму (17) проводилась при попередніх початкових даних. Нижче наведені результати обчислювальних розрахунків. В табл. 3 дано початковий закон руху $v^{0}(t)=\left(v_{1}^{0}(t), v_{2}^{0}(t)\right)$ рухомого граничного джерела. Оптимальний закон руху $v^{*}(t)=\left(v_{1}^{*}(t), v_{2}^{*}(t)\right)$ рухомого регулятора (10), отриманий за алгоритмом (17), подано в табл. 4. 
Таблиия 3

\begin{tabular}{|c|c|c|}
\hline$t$ & $v_{1}^{0}(t)$ & $v_{2}^{0}(t)$ \\
\hline 0,0 & 0,0 & 0,0 \\
\hline 0,02 & 0,667 & 0,0 \\
\hline 0,04 & 1,333 & 0,0 \\
\hline 0,06 & 2,0 & 0,0 \\
\hline 0,08 & 2,0 & 0,5 \\
\hline 0,10 & 2,0 & 1,0 \\
\hline 0,12 & 1,333 & 1,0 \\
\hline 0,14 & 0,667 & 1,0 \\
\hline 0,16 & 0,0 & 1,0 \\
\hline 0,18 & 0,0 & 0,5 \\
\hline
\end{tabular}

Таблиия 4

\begin{tabular}{|c|c|c|}
\hline$t$ & $v_{1}^{*}(t)$ & $v_{2}^{*}(t)$ \\
\hline 0,0 & 0,010 & 0,0 \\
\hline 0,02 & 0,765 & 0,0 \\
\hline 0,04 & 1,230 & 0,0 \\
\hline 0,06 & 1,990 & 0,0 \\
\hline 0,08 & 2,0 & 0,5 \\
\hline 0,10 & 1,990 & 1,0 \\
\hline 0,12 & 1,285 & 1,0 \\
\hline 0,14 & 0,664 & 1,0 \\
\hline 0,16 & 0,010 & 1,0 \\
\hline 0,18 & 0,0 & 0,5 \\
\hline
\end{tabular}

Значення функціонала (16) при цьому зменшилось $3 \quad J\left(z^{0}\right)=0,639538$ до $J\left(z^{*}\right)=0,438419$. На рис. 2 зображено оптимальне керування (10), рух якого здійснюється по оптимальній траекторії, наведеній в табл. 4. Оптимальна траекторія складається 3 чотирьох частин, кожна 3 яких нагадує параболу i визначає (описує) рух регулятора вздовж відповідної границі прямокутної області.

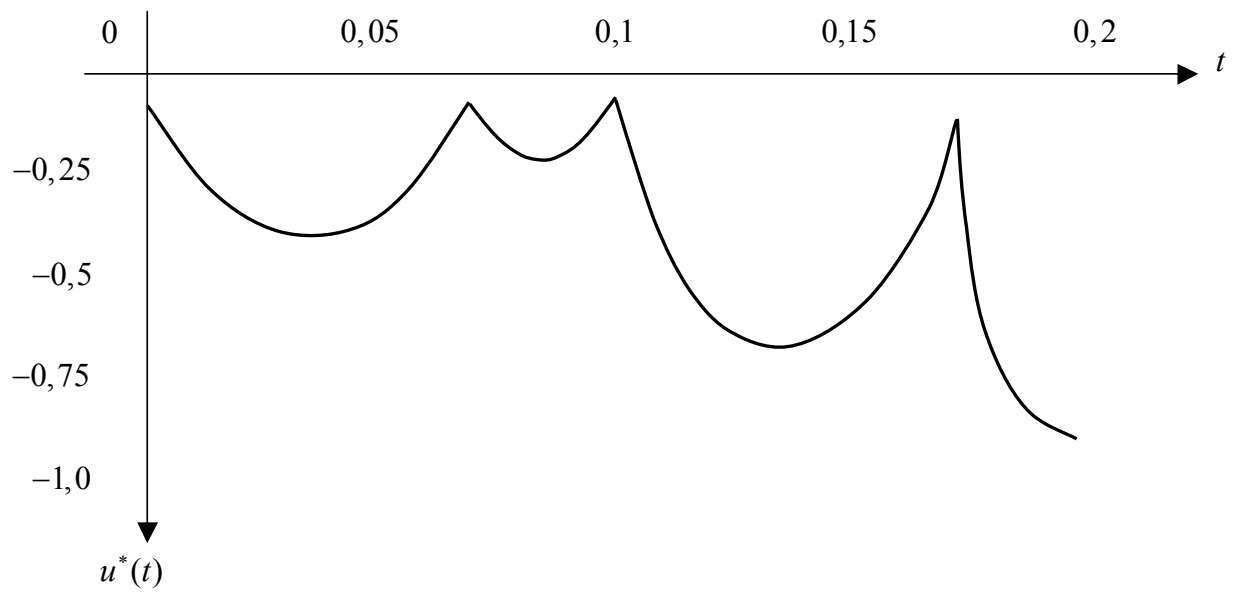

Рис. 2. Графік оптимального граничного рухомого керування

Обчислювальні експерименти також показали, що ефективність точкових і рухомих граничних керувань зростає із зменшенням коефіцієнта температуропровідності $a$, тобто із зменшенням цього коефіцієнта значення функціонала (8) після визначення оптимальної стратегії керування зменшувалось на більшу величину порівняно із значенням цього ж функціонала при заданій початковій стратегії керування.

\section{Висновки}

Результатом дослідження $є$ розв'язок задачі оптимального розташування точкових граничних регуляторів та задачі визначення оптимальної траєкторії 
переміщення рухомого регулятора по границі області, в якій функціонує розподілений об'єкт керування. Задача розв'язана в мінімаксній постановці, тобто знайдено оптимальний регулятор за станом об'єкта, який функціонує в умовах невизначеності, причому збурення об'єкта належать заданій обмеженій області. Наведено результати обчислювальних експериментів, які ілюструють ефективність побудованих зосереджених граничних точкових та рухомих регуляторів. Отримані результати свідчать про те, що знайдені керування дійсно є оптимальними і забезпечують мінімум похибки (відхилення від заданого стану) функціонування системи та енергетичних затрат на здійснення керування при заданих умовах і відсутності будь-якої інформації про зовнішній вплив, крім області допустимих збурень. Задовільні показники якості керування спостерігаються навіть за умов виходу збурень за межі заданої області.

\section{Література}

1. Бутковский А.Г. Методи управления системами с распределенными параметрами / А.Г. Бутковский. — Москва :Наука, 1975. - 568 с.

2. Бутковский А.Г. Подвижное управление системами с распределенными параметрами / А.Г. Бутковский, Ю.В. Даринский, Л.М. Пустыльников. - Автоматика и телемеханика, 1976. - № 2. - С. 15-25.

3. Бутковский А.Г. Теория подвижного управления сисистемами с распределенными параметрами / А.Г. Бутковский, Л.М. Пустыльников. — Москва :Наука, 1980. — 397 с.

4. Лионс Ж.-Л. Оптимальное управление системами, описывающимися уравнениями с частными производными / Ж.-Л. Лионс. — Москва :Мир, 1972. — 414 с.

5. Ладыженская O.A. Линейные и квазилинейные уравнения параболического типа / О.А. Ладыженская, Н.Н. Уральцева, В.А. Солонников. - Москва :Наука, 1967. — 736 с.

6. Лобок О.П. Мінімаксне управління в лінійних динамічних системах із розподіленими параметрами / О.П. Лобок, Б.М. Гончаренко, Н.М. Савіцька // Журнал «Наукові праці Національного університету харчових технологій». — Том 21, № 6. - Київ : НУХТ. 2015. - C.16-26.

7. Кириченко Н.Ф. Минимаксное управление и оценивание в динамических системах / Н.Ф. Кириченко. — Автоматика и телемеханика, 1982. — № 1. - С. 32-39.

8. Лобок А.П. Минимаксные регуляторы в системах с распределенными параметрами. Вестн. Киев. Универ. Моделирование и оптимизация сложных систем. — 1983. — вып. 2. — C. $62-67$.

9. Васильев Ф.П. Методы решения экстремальных задач / Ф.П. Васильев. — Москва : Наука, 1981. - $400 \mathrm{c}$. 